\title{
Antibiotics Prescribing Pattern at Outpatient Department of A Tertiary Medical College Hospital
}

\author{
Maliha Ata ${ }^{1 *}$ \\ Rozina Hoque ${ }^{1}$ \\ Rajat Shankar Roy Biswas ${ }^{2}$ \\ Asma Mostafa ${ }^{3}$ \\ Faheem UI Hasan ${ }^{4}$ \\ Happy Rani Barua ${ }^{1}$
}

${ }^{1}$ Department of Pharmacology

Chattagram Maa-O-Shishu Hospital Medical College Chittagong, Bangladesh

${ }^{2}$ Department of Medicine

Chattagram Maa-O-Shishu Hospital Medical College Chittagong, Bangladesh

${ }^{3}$ Department of Anatomy

Chattagram Maa-O-Shishu Hospital Medical College Chittagong, Bangladesh

${ }^{4}$ Department of Community Medicine Chattagram Maa-O-Shishu Hospital Medical College Chittagong, Bangladesh

\section{*Correspondence to:}

\section{Dr. Maliha Ato}

Associate Professor

Department of Pharmacology

Chattagram Maa-O-Shishu Hospital Medical College

Chittagong, Bangladesh.

Mobile : +8801758 000927

Email:dr.maliha59@gmail.com

www.banglajol.info/index.php/CMOSHMCJ

\begin{abstract}
Background: In order to improve the prescription quality and promoting rational prescription pattern, there is an obligatory need to investigate the factors that affect doctors' prescription patterns. The study was conducted to observe the antibiotics prescribing pattern at outpatient department of a tertiary medical college hospital. Methods: This was a descriptive cross sectional study in a tertiary medical college hospital during the period of January 2018 to June 2018 which was conducted on 300 prescriptions collected from different outpatient department. Results: Average number of drugs prescribed per encounter was 3.70 (Optimal value 1.6-1.8). Antibiotics (Oral, injectable and topical) were prescribed 46\% (Optimal value 20.0-26.8\%) and antibiotic as only injected form $19.71 \%$ (Optimal value $13.4-24.1 \%$ of total injectable drug). Drugs prescribed from the Essential Drugs List( EDL) equated to $52.90 \%$ (Optimal value 100\%). The antibiotics given in most of the patients (91\%) were without doing culture sensitivity test before prescribing. Out of $46 \%$ prescriptions with antibiotics, $79.9 \%$ had one antibiotic, $19.6 \%$ included two antibiotics and $0.7 \%$ had three antibiotics. Cefuroxime was the most commonly prescribed antibiotics (22.5\%) followed by Azythromycin (11.6\%) Cefixime (11.5\%) Ciprofloxacin (10.9\%) Flucloxacillin (10.9\%) and Metronidazole (8.7\%). Conclusion: This study revealed that percentage of antibiotic was high and most of the antibiotic was given without culture and sensitivity.
\end{abstract}

Key words: Antibiotic; Essential Drug List; Rational; Prescription.

\section{INTRODUCTION}

Nowadays antibiotic is the most commonly prescribed drug in hospitals, worldwide ${ }^{1}$. Lack of strict regulation on the excessive and inappropriate use of antibiotic leading to increased drug resistance ${ }^{2}$. So the rational use of antibiotics is a serious challenge and become a major health need to the health care professionals against development of resistance ${ }^{3}$. Some factors that contribute to the development of antibiotic resistance-antibiotics overprescribing even for viral infections, overusage and incomplete duration of antibiotics, over the-counter availability of antibiotics, inadequate patient counseling, and patients buying only as many tablets as they can afford ${ }^{4}$. Sometimes doctors claimed to prescribe antibiotic as per patient's demand ${ }^{5}$. In 2015, the White House released The National Action Plan for Combating Antibiotic-Resistant Bacteria (CARB) which set a goal of reducing inappropriate outpatient antibiotic use by at least half by $2020^{6}$. National Ambulatory Medical Care Survey (NAMCS) and the National Hospital Ambulatory Medical Care Survey (NHAMCS) in the United States from 2010-2011, found that the estimated 154 million prescriptions for antibiotics written in doctor's offices and emergency departments each year, among them 30 percent are unnecessary. This finding creates a benchmark for improving outpatient antibiotic prescribing and use for the developed country ${ }^{7}$. In developing country like Bangladesh, $55.57 \%$ of the 
doctors prescribe antibiotics in suspected infection while only $33.46 \%$ of them prescribe antibiotics in confirmed cases. 40.22 $\%$ of doctors prescribe antibiotics in cold and fever before any diagnostic test. Moreover, $37.31 \%$ of doctors prescribe antibiotics for pleasing the patients whereas $62.44 \%$ denied such undue influence. Doctors occasionally receive feedback from patients on completion of course of antibiotic therapies ${ }^{8}$. The study of prescribing pattern infers to monitor, assess and conceive modifications in the practitioner's prescription habits to ensure reasonable and effective care of the patient ${ }^{9}$. The knowledge about antibiotic utilization patterns is essential for an organized approach to problems that arise from multiple antibiotic usages. It is extremely important that institutions and hospitals should have an antibiotic policy so that the best choices are made by individual prescribers ${ }^{10}$. Disposition of evidence based care on regulated antibiotic prescribing can be acquired through periodic prescription audit ${ }^{11}$. Monitoring antibiotic prescriptions can provide feedback about prescription patterns to the physicians ${ }^{12}$. By considering all the facts described above, this study was aimed to describe the current pattern of antibiotic use in outpatient departments in a Tertiary Medical College Hospital in Chittagong.

\section{MATERIALS AND METHODS}

This was a descriptive cross sectional study in a Tertiary Medical College Hospital during the period of January 2018 to June 2018. A total of 300 prescriptions from different outdoor were used as sample by convenient sampling. For data collection, a self designed data collection sheet was used. After pretesting the tool, data collectors collected different outdoor prescription from exit point of hospital outdoor convenient to their time and patient approach. They informed the patient about the study and took permission to capture the photograph of the prescription and Culture and Sensitivity $(\mathrm{C} / \mathrm{S})$ test report related to that prescription. After collecting the photograph of each prescription and related $\mathrm{C} / \mathrm{S}$ report, data was put in the data collection sheet. Patient of less than 15 years and patient who was going to admitted to the hospital and patient who was not willing to participate in the study were excluded from the study. After completing 300 prescriptions, data was put in the data collection sheet.

Collected data were analyzed by SPSS 18 and descriptive statistics such as frequencies and percentages were used in the analysis of the data.

\section{RESULTS}

Three hundred prescriptions were included in the study. Antibiotic (Oral, injectable and topical) prescribed in total 138 (46\%) prescriptions among which male 51(36\%) and female $87(63 \%)$ and antibiotic not prescribed in 162 (54\%) prescriptions (Fig 1). Among there hundred prescription 119 (40\%) prescriptions were prescribed by consultant and 181 $(60 \%)$ prescription were prescribed by nonconsultant. A high number of prescriptions in which the diagnosis (81\%) were not written and about $94 \%$ antibiotic were prescribed without culture and sensitivity testing. Average number of the drug per prescription 3.70. Antibiotic prescribed by essential drug list 21 out of $138(15.22 \%)$. Out of the surveyed antibiotic prescriptions, $79.9 \%$ contained one antibiotic, $19.6 \%$ contained two antibiotics, and $0.7 \%$ comprised of three antibiotics. Among 138 prescribed antibiotics, $19.71 \%$ was prescribed in injectable form and $18.8 \%$ were prescribed in topical form. The study also assessed the type of antibiotics prescribed (Table 1).

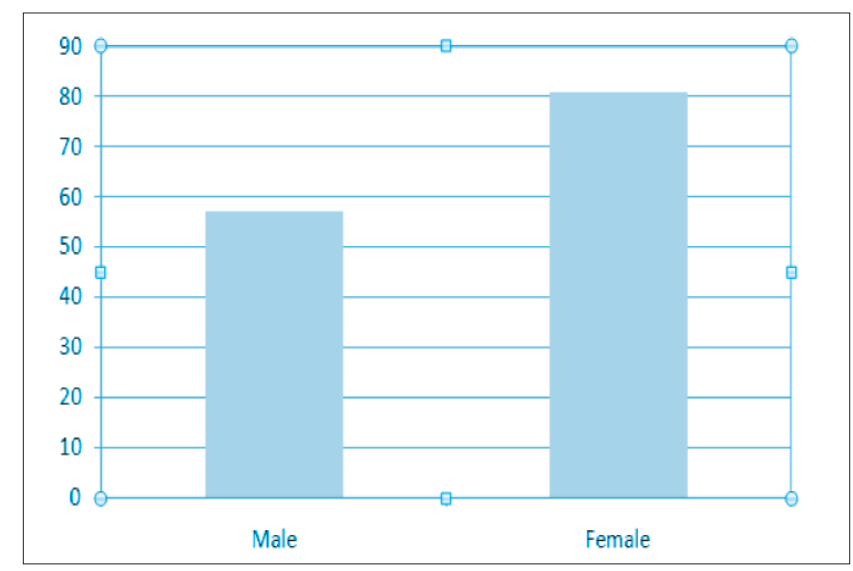

Figure 1: Sex distribution according to the presence of antibiotic

Table 1 : Prescribing indicators used $(n=300)$

$\begin{array}{ll}\text { Prescribing Indicators } & \text { Numbers of drugs/percentage } \\ \text { Total number of the prescription analyzed } & 300 \text { cases } \\ \text { Average drug encountered per patient } & 3.70 \text { (Optimal value: } 1.6-1.8) \\ \text { Total number of drugs prescribed } & 1110 \\ \text { Total number of antibiotics } & 138(46 \%)(\text { Optimal } \\ \text { encountered in the study } & \text { value: 20.0-26.8\%) } \\ \text { Number of antibiotics given as Monotherapy } & 79.9 \% \\ \text { Two antibiotic prescribed per prescription } & 19.6 \% \\ \text { Three antibiotic prescribed per prescription } & 0.7 \% \\ \text { Antibiotic prescribed by Essential drug list } & 52.90 \% \text { (Optimal value: 100\%) } \\ \text { Number of antibiotics prescribed with an injection } & 19.71 \% \\ \text { Number of antibiotics prescribed with topical form } & 18.8 \%\end{array}$

Out of 138 antibiotics prescribed in the study, $22.5 \%$ were of Cefuroxime, $11.6 \%$ were of Azythromycin, 11.5\% were of Cefixime, $10.9 \%$ were of Ciprofloxacin, $10.9 \%$ were of Flucloxacillin, $8.7 \%$ were of Metronidazole,7.2\% were of Moxifloxacin and Mupiricin, 5.8\% were of Cefuroxime + Clavulonic acid, $4.3 \%$ were of Ceftriaxone, $2.9 \%$ were of Levofloxacin and Clindamycin, $2.5 \%$ were of Nitrofurantoin, $2.2 \%$ were of Cefaclor and Tobramycin, $1.4 \%$ Amoxicillin+ Clavulonic acid, $0.7 \%$ were of Cefradine, Gentamicin and amoxicillin antibiotics ( Fig 2). 


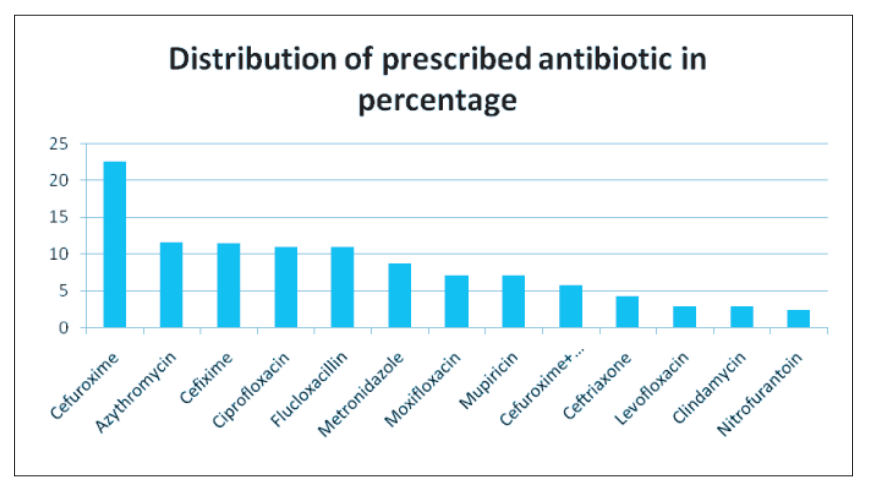

Figure 2 : Distribution of prescribed antibiotics in percentage

\section{DISCUSSION}

Antibiotics are the most commonly prescribed drugs in hospitals in developing country. Majority of the patients in hospitals obtain antibiotics without $\mathrm{C} / \mathrm{S}$ test. Prescribing antibiotics haphazardly can lead to increased antibiotic resistance and the scenario is more common in developing nations. A prescription based survey is considered to be one of the most effective method to assess and evaluate the prescribing attitude of physician ${ }^{13}$. This study was carried out with the purpose to determine the prescribing pattern of antibiotics among the physicians of a Tertiary Medical College outdoor in Chittagong. The average number of drugs per prescription was found to be 3.70 in our study which was less than that reported from studies conducted in India 2016 (7) and more than in Yemen 2016 (3.2) and Pakistan 2011(3.13) but slightly higher than the recommended limit by the $\mathrm{WHO}^{14-18}$. In this study, $46 \%$ prescriptions containing antibiotics which is very less than that of the another study in Dhaka city private practitioner $(70.33 \%)$ and also less than that obtained from Pakistan (52.4\%) Jordan (85\%) but more than WHO prescribing indicator ${ }^{20-21}$. According to WHO, $15-25 \%$ of antibiotics encountered is expectable in the countries where an infectious disease is more prevalent ${ }^{22}$. In a 3rd world developing country like Bangladesh, prevalence of infectious diseases is higher than the developed countries. That is why in this study the antibiotic utilization rate was higher than that of developed countries. The disease condition for which antibiotic were prescribed could not be measured as prescription collected from different outdoor and maximum (81\%) prescription contain no diagnosis. Most (79.9\%) of the prescriptions contained single antibiotic which is good indicator of prescribing pattern of antibiotics in terms of number of antibiotic per prescription. Only $52.9 \%$ of the prescribed antibiotics were from the Essential Drug List (EDL) of WHO. Most commonly used prescribed antibiotic was Cefuroxime (22.5\%) which is excluded from the Essential Drug List (EDL) of WHO. Prescribing from the EDL is good clinical practice and improves the rational use of medicines. Similar study conducted at Madhya Pradesh India where it was $66.9 \%$, West Bengal, India where it was $90.3 \%$ and in Nigeria 94\% drugs prescribed from EDL which was very higher as compared to our study while from Srilanka 39.6\% drugs prescribed from EDL which was lower as compared to our study.
Similar studies were also done in Dhaka, Bangladesh where it was $46.31 \%$ which was also lower as compared to our study ${ }^{19,23-26}$. In study of outpatient department of a tertiary medical college hospitals of Bangladesh 2011, Cefuroxime $(3.33 \%)$ was least commonly used antibiotic but now in our study which is done in 2108 , Cefuroxime $(22.5 \%)$ is the most commonly used antibiotic in outpatient department ${ }^{27}$. There can be various factors behind this such as, may be infection prevalence more in our country, prescribers lacking of the understanding the importance of essential drug concept, influence from the manufacturers to promote a specific brand ${ }^{28-29}$. In our study $19.71 \%$ antibiotic prescribed as injectable form which is less than the number stated in Yemen $(27.4 \%)^{15}$. But this rate is high as the WHO recommended target for injection exposure is $10 \%$ or $\operatorname{less}^{21}$.

\section{LIMITATIONS}

The analysis of antibiotic prescriptions was not based on diagnosis pattern. Antibiotic prescriptions from specific groups like pregnant women, children and geriatric patients were not analyzed and the cost of the antibiotics were not calculated. Moreover it is done for a very short period of time and with limited amount of prescriptions. The prescription practices may have changed as a result of seasonal variation.

\section{CONCLUSION}

This study revealed some deviation from rational prescribing by the prescribers because average number of drugs per prescription was higher than that of the recommended by the WHO, antibiotic prescription was considerably higher and maximum antibiotic prescribed without culture and sensitivity testing. Most commonly used antibiotic (Cefuroxime) is out of the Essential Drug list of the WHO. In our study maximum prescription contain single antibiotic which is one of a good indicator of rational prescribing. To create awareness of the rational use of antibiotics is compulsory, basically aimed at reducing the overall prescribing of antibiotics and encouraging to start with a narrower spectrum.

\section{DISCLOSURE}

All the authors declared no competing interest. 


\section{REFERENCES}

1. Rubin RP. A brief history of great discoveries in pharmacology: In celebration of the centennial anniversary of the founding of the American Society of Pharmacology and Experimental Therapeutics. Pharmacol Rev. 2007; 59:289-359.

2. Faryna A, Gilbert L, Wergowske, Goldenberg K. Impact of therapeutic guidelines on antibiotic use by residents in primary care clinics. J Gen Intern Med. 1987; 2:102-107.

3. Ahmad A, Khan MU, Moorthy J, Jamshed SQ, Patel I. Comparison of knowledge and attitudes about antibiotics and resistance, and antibiotics self-practicing between Bachelor of Pharmacy and Doctor of Pharmacy students in Southern India. Pharm Pract. 2015; 13(1):523.

4. University of Washington, School of Public Health and Community Medicine. 2000. Antibiotic Resistance. http://depts.washington.edu/eminf/2000/resistance/resist2.html.

5. Sivagnanam G, Thirumalaikolundusubramanian P, Mohanasundaram J, Raaj AA, Namasivayam K, Rajaram S. A Survey on Current Attitude of Practicing Physicians Upon Usage of Antimicribial Agents in Southern Part of India. Medscape General Medicine .2004; 6(2):1.

6. National action plan for combating antibiotic resistance bacteria. The White House, Washington. 2015.

7. Centers for Disease Control and Prevention: 1 in 3 antibiotic prescriptions unnecessary. Available at: https://www.cdc.gov/media/releases/2016/p0503-unnecessary-prescriptions.html

8. Hasan SMR, Hossain MM, Akter R, Karim SMH, Haque S, Kamaluddin M, Ghani A. Pattern of Antibiotics Use at the Primary Health Care Level of Bangladesh: Survey Report-1. S. J. Pharm. Sci.2009; 2(1): 1-7.

9. Shankar RP, Partha P, Shenoy NK, Easow JM, Brahmadathan KN. Prescribing patterns of antibiotics and sensitivity patterns of common microorganisms in the Internal Medicine ward of a teaching hospital in Western Nepal: A prospective study. Ann Clin Microbiol Antimicrob. 2003; 2:7

10. National policy for containment of antimicrobial resistance [Internet]. New Delhi: Directorate General of Health Sciences. https://mohfw.gov.in/sites/default/files/3203490350abpolicy\%20\%281\%29.pdf.

11. Harbarth S, Harris AD, Carmeli Y, Samore MH. Parallel analysis of individual and aggregated data on antibiotic exposure and resistance in gram-negative bacilli. Clin Infect Dis. 2001; 33(9):1462-1468.

12. Srishyla MV, Naga Rani MA, Venkataraman BV. Drug utilization of antimicrobials in the in- patient setting of a tertiary hospital. Indian J Pharmacol. 1994; 26:282-287.

13. Vlahovic-Palcevski V, Morovic M, Palcevski G. Antibiotic utilization at the university hospital after introducing an antibiotic policy. Eur J Clin Pharmacology. 2000; 56:97-101.

14. Pallavi PS, Sree BT and Krishnakanth PV. Study of prescription patterns of antibiotics in tertiary care hospital. International Journal of Biomedical Research. 2016; 7(6): 372-374.

15. Alshakka M, Said K, Babakri M, Ansari M, Aldhubhani A, Hassali MA, Ibrahim MIM. A Study on Antibiotics Prescribing Pattern at Outpatient Department in Four Hospitals in Aden-Yemen . Journal of Pharmacy Practice and Community Medicine. 2016 ; 2(3):88-93.

16. Riaz H, Malik F, Raza A, Hameed A, Ahmed S, Shah PA, Hussain S. Assessment of antibiotic prescribing behavior of consultants of different localities of Pakistan. African Journal of Pharmacy and Pharmacology.2011; 5(5): 596-601.

17. Hogerzeil HV, Bimo, Ross-Degnan D, Laing RO, Ofori-Adjei D, Santoso B. Field tests for rational drug use in twelve developing countries. Lancet.1993; 342(8884):1408-1410.

18. World Health Organization. 2001. Interventions and Strategies to Improve the Use of Antimicrobials in Developing Countries: Drug Management Program. Geneva: WHO; (WHO/CDS/CSR/DSR/2001.9.

19. Begum F, Uddin MR, Islam MMS, Sarker MN, Barman RC, Ali MY. Evaluation of Prescribing Pattern of the Private Practitioners in Bangladesh.Faridpur Med. Coll. J. 2012;7(2):51-53.

20. Atif M, Azeem M, Sarwar MR, Shahid S, Javaid S, Ikram H, Baig U, Scahill S. WHO/INRUD prescribing indicators and prescribing trends of antibiotics in the Accident and Emergency Department of Bahawal Victoria Hospital, Pakistan. SpringerPlus. 2016 ; 5:1928.

21. Al-Niemat SI, Aljbouri TM, Goussous LS, Efaishat RA, Salah RK. Antibiotic prescribing patterns in outpatient emergency clinics at Queen Rania Al Abdullah II Children's Hospital, Jordan. Oman Med J. 2013;29(4):250.

22. World Health Organization (WHO). International Network for Rational Use of Drugs and World Health Organization. How to investigate drug use in health facilities: Selected drug use indicators 1993. EDM Research Series No. 7 [WHO/DAP/93.1].

23. Bhartiy SS, Shinde M, Nandeshwar S, Tiwari SC. Pattern of prescribing practices in the Madhya Pradesh, India. Kathmandu University Medical Journal.2008;6(1):55-59.

24. Dutta A, Chakraborty S. Practice of rational drug uses in a rural area of 24 pgs (s) in West Bengal. Journal of Advanced Pharmaceutical Technology \& Research. 2010;1(3):358-364.

25. Tamuno I, Fadre J. Drug utilization pattern in a Nigerian tertiary hospital. Trop J Pharm Res. 2012; 11:146-152

26. Prasad K, Ranasinghe B. Pattern of private sector drug prescription in Galle: A descriptive cross-sectional study. Gall Med J. 2006 ;64-68.

27. Alam MM, Parveen F, Ara F, Iqbal MJU, Saha RR. Prescribing Trends in the Out Patient Department in a Tertiary Hospital in Bangladesh. Bangladesh Medical Journal. 2011; 40(2):8-11.

27. Kotwani A, Wattal C, Katewa S, Joshi PC, Hollowy K. Factors influencing primary care physicians to prescribe antibiotics in Delhi India. Family Practice. 2010; 27(6):684-690.

28. Goyal R, Pareek P. A Review article on prescription behavior of doctors, influenced by the medical representative in Rajasthan, India. IOSR J Busin Manage. 2013;8(1):56-60. 